\title{
ARE WE SCREENING TOO MANY BABIES FOR RETINOPATHY OF PREMATURITY?
}

\author{
RICHARD R. GOBLE ${ }^{1}$, HELEN S. JONES ${ }^{2}$ and ALISTAIR R. FIELDER ${ }^{2}$ \\ Birmingham
}

\begin{abstract}
SUMMARY
A retrospective, hospital-records-based study of neonates screened for retinopathy of prematurity (ROP) was undertaken to determine whether the inclusion criteria for screening could have been safely altered to reduce the numbers of babies screened whilst not missing any stage III disease. Babies from six neonatal intensive care units in Birmingham were screened by a single examiner. Between November 1989 and November 1995, 1611 babies were examined and 1429 of these fell within the inclusion criteria of current guidelines for ROP screening produced by the Royal College of Ophthalmologists and the British Association of Perinatal Medicine - any baby $\leqslant 1500 \mathrm{~g}$ birthweight or $\leqslant 31$ weeks gestational age. Thirty-nine (39) babies developed stage III ROP of which $31(2.2 \%)$ were from Birmingham. In addition 8 babies with stage III ROP were referred from elsewhere. All 39 babies with stage III ROP had a birthweight $\leqslant 1250 \mathrm{~g}$ or a gestational age of $\leqslant 29$ weeks, but 2 fell outside one criterion, indicating the need for both to be used. Had these inclusion criteria been utilised during this period, then $30 \%$ fewer babies would have been examined (432 of 1429). The importance of using both birthweight and gestational age as inclusion criteria is discussed, and the dangers of altering the indications for national screening on the basis of one study population is emphasised.
\end{abstract}

Screening for retinopathy of prematurity (ROP) is difficult to learn, time consuming and potentially hazardous for the baby. However, by this process severe disease is identified and treated. Although the proportion of treated cases to those screened is small, outcome is significantly improved ${ }^{1}$ and each successful case represents many years of prevented blindness. It is for this reason that screening has been

From: ${ }^{1}$ Birmingham and Midland Eye Hospital, Birmingham; ${ }^{2}$ Department of Ophthalmology, University of Birmingham Medical School, Birmingham, UK.

Correspondence to: Professor Alistair Fielder, FRCP, FRCS FRCOphth, Academic Unit of Ophthalmology, Imperial College School of Medicine at St Mary's, The Western Eye Hospital, 171 Marylebone Road, London NW1 5YE, UK. Tel: +44 (0)171 402 4211. Fax: +44 (0)171258 0133 . shown to be effective in a financial scenario as well as in clinical terms. ${ }^{2}$

The most recent guidelines for ROP screening from the Royal College of Ophthalmologists and the British Association of Perinatal Medicine ${ }^{3}$ include the following inclusion criteria: any baby $\leqslant 1500 \mathrm{~g}$ birthweight (BW) or $\leqslant 31$ weeks gestational age (GA). Screening programmes are designed to ensure that no baby with severe disease remains unidentified. This is balanced against the importance of keeping the workload to a minimum by targeting only the population at risk. To this end we have undertaken a retrospective study of ROP screening in Birmingham to assess whether the current guidelines are appropriate or include many babies who are not at risk of developing severe disease. The specific aim of this study was to determine which babies are at risk of severe disease, which we defined as any stage III. We chose this end point for two reasons: first, there is a distinct possibility that the threshold for treatment will be modified in the near future to include milder degrees of stage III ROP. Second, as babies with stage III ROP have a high risk of ophthalmic sequelae they require periodic review. ${ }^{4,5}$

\section{PATIENTS AND METHODS}

A retrospective analysis of ROP screening records between November 1989 and November 1995 was undertaken. The notes from all six neonatal intensive care units in Birmingham were reviewed. The following data was collected: BW, GA, incidence of stage III ROP, and whether treatment was employed. All babies with a $\mathrm{BW} \leqslant 1700 \mathrm{~g}$, or $\leqslant 32$ weeks GA were included in this screening programme. Examinations, by a single observer (A.R.F.), were performed mainly on a weekly basis, although sometimes fortnightly, commenced at 3 weeks postnatal age and continued if normal until vascularisation had reached zone 3 . Those with ROP were reviewed as clinically indicated. Pupil dilatation was achieved using $0.5 \%$ cyclopentolate and $2.5 \%$ phenylephrine eyedrops instilled once at least 30 minutes before examination. Indirect 


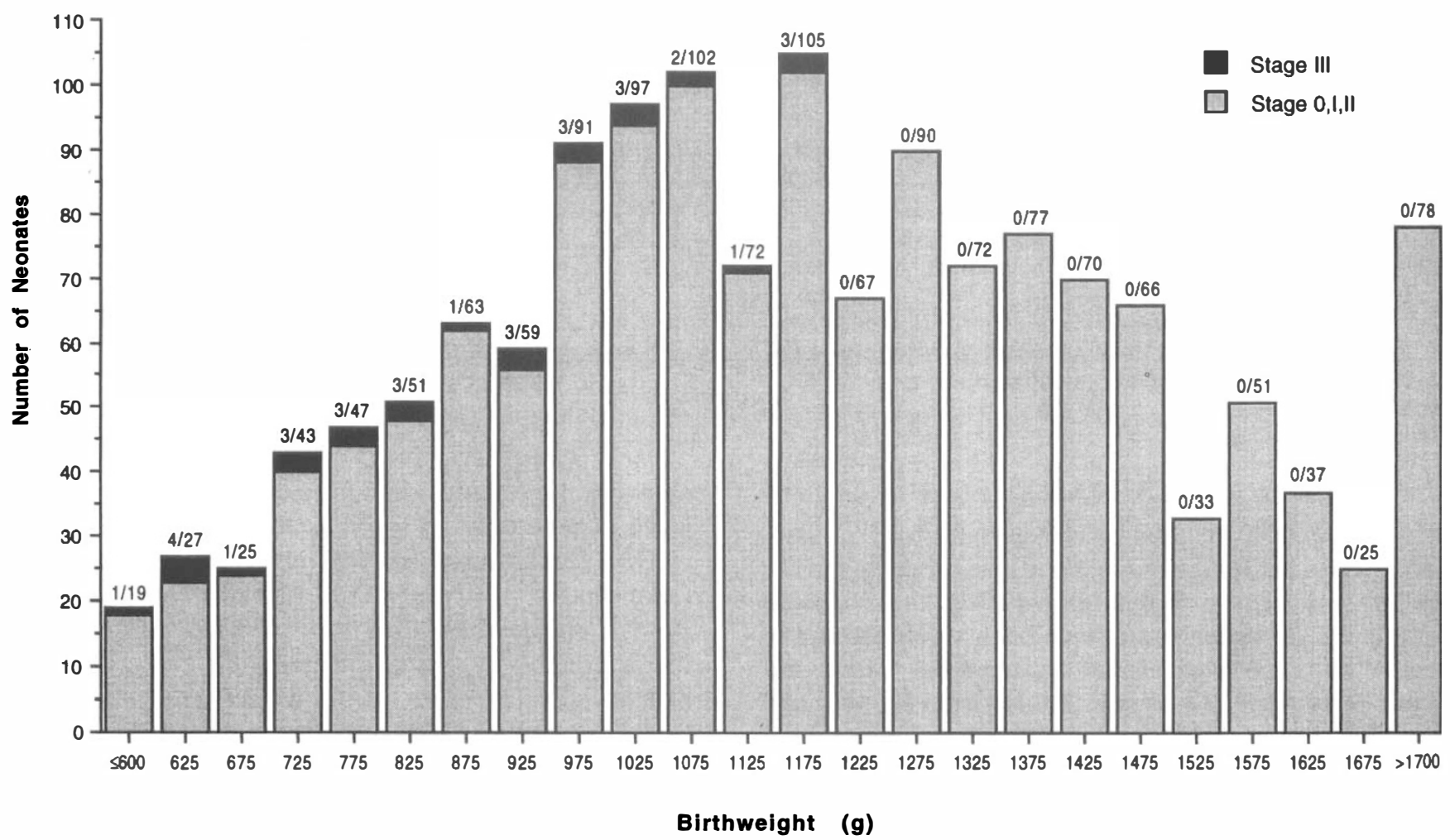

Fig. 1. Birthweights of 1427 Birmingham neonates screened for ROP. Midpoint values of birthweight groups (apart from the first and last) are given.

ophthalmoscopy was performed using a 28 dioptre lens and lid speculum after application of topical anaesthetic ( $0.4 \%$ oxybuprocaine). A scleral indentor was routinely used for ocular rotation rather than indentation. ${ }^{6}$ Details of each examination were recorded using the International Classification of ROP. $^{7,8}$

\section{RESULTS}

Birmingham Babies Screened for ROP

Of the total 1611 babies screened, 182 fell outside the current clinical guidelines: $\leqslant 1500 \mathrm{~g} \mathrm{BW}$ or $\leqslant 31$ weeks GA. None of these larger 182 babies developed stage III ROP. Of the remaining 1429 babies, $31(2.2 \%)$ developed stage III ROP.

BW measurements were obtained in 1427 of the 1429 (99.9\%). The average BW was $1199 \mathrm{~g}$ (SD \pm 312 , range $465-3080 \mathrm{~g}$ ). The $\mathrm{BW}$ distribution is shown in Fig. 1; no baby $>1200 \mathrm{~g}$ developed stage III ROP. GA data were available in 1423 (99.6\%). The mean GA was 29.1 weeks (SD \pm 2.1 , range $24-38$ weeks). The distribution of GA is shown in Fig. 2; the highest GA associated with stage III ROP was 29 weeks.

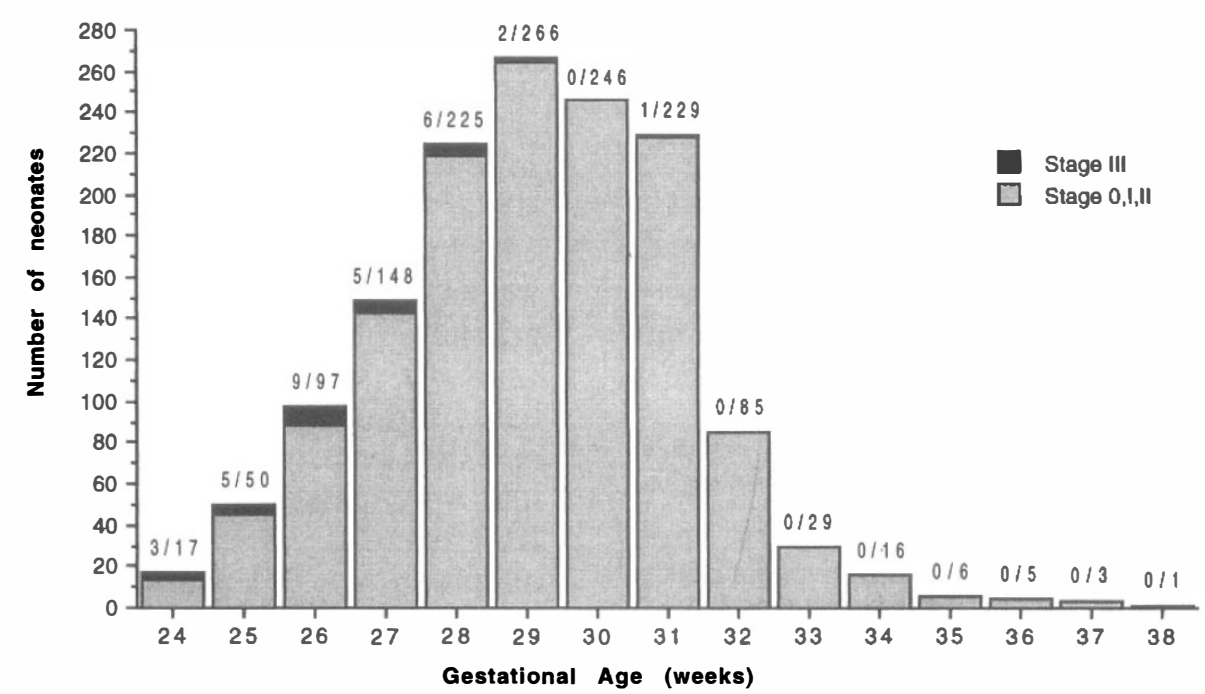

Fig. 2. Gestational ages of 1423 Birmingham neonates screened for ROP. 


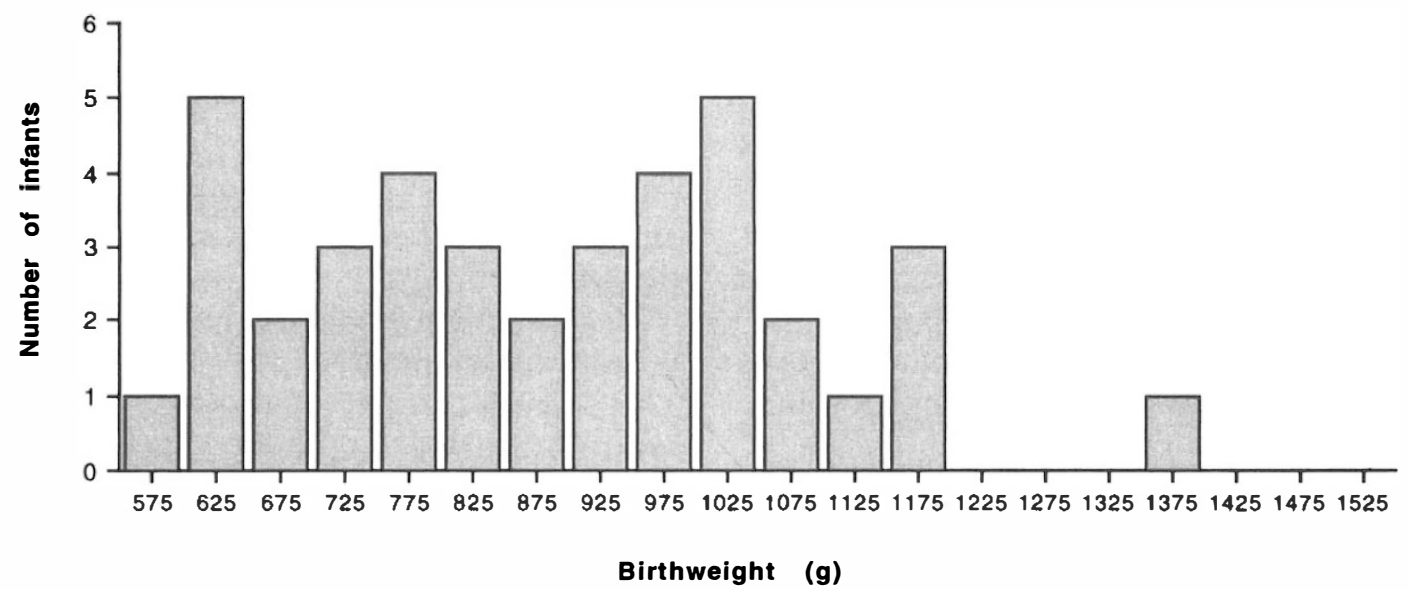

Fig. 3. Birthweights of all 39 neonates with stage III ROP. Midpoint values of birthweight groups are given.

\section{Stage III Babies}

Of the 39 babies diagnosed with stage III disease, 31 were born in Birmingham and 8 were referred from other areas. Twenty-four (24) neonates were treated: 17 from Birmingham and 7 from elsewhere. The BW and GA data for this group of 39 stage III neonates is shown in Figs. 3 and 4. No baby presented with stage IV or V disease. The mean GA and BW of treated and non-treated stage III babies was respectively $26.3(\mathrm{SD}=1.9)$ weeks and $26.7(\mathrm{SD}=1.2)$ weeks, and $937(\mathrm{SD}=185.9) \mathrm{g}$ and $874(\mathrm{SD}=183.4) \mathrm{g}$.

The two largest/most mature babies with stage III ROP were: $1375 \mathrm{~g} \mathrm{BW}$ and 29 weeks GA, and $930 \mathrm{~g}$ $\mathrm{BW}$ and 31 weeks GA.

\section{DISCUSSION}

The population presented here is particularly suitable for scrutinising the criteria for ROP screening. First, the Birmingham criteria included babies who were larger and more mature than recommended by the current guidelines. ${ }^{3}$ Second, while not as rigorous as a previous epidemiological study, ${ }^{9}$ the protocol,

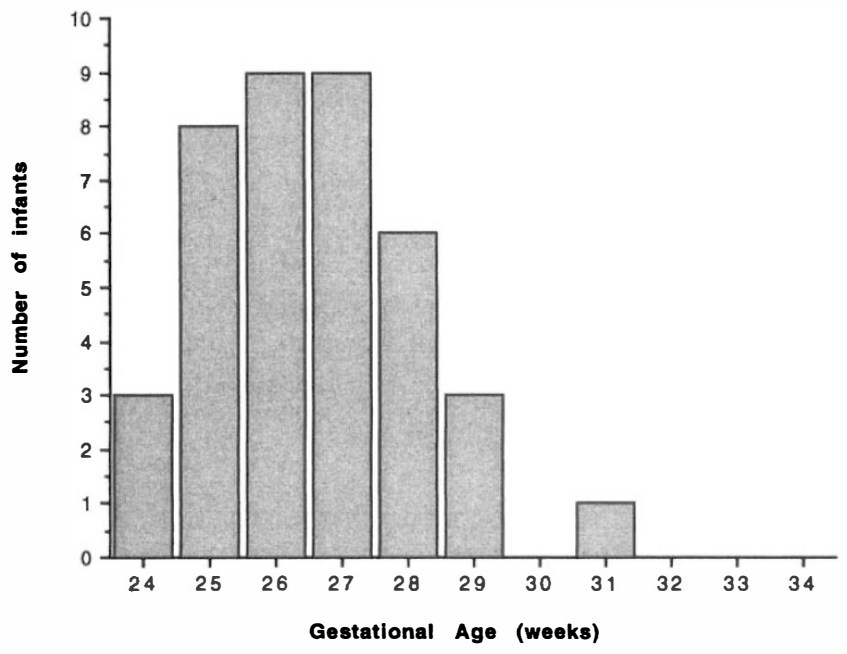

Fig. 4. Gestational ages of all 39 neonates with stage III $R O P$. with weekly examinations, commencing at 3 weeks, was more intensive than most in routine clinical practice. Both factors served to minimise the possibility that any severe ( $\geqslant$ stage III ROP) was missed.

The overall incidence of stage III disease, in this population of babies from Birmingham, was $2.2 \%$. These results are now compared with several recent studies (data from the literature in parentheses). ${ }^{9-19}$ Not every study cited contributes to each category. By BW, stage III ROP was observed: $<750 \mathrm{~g}$, in $9.6 \%$ $(25.0-70.0 \%) ; \quad 750-999 \mathrm{~g}, \quad 4.6 \% \quad(19.0-32.3 \%)$; $1000-1250 \mathrm{~g}, 2.0 \%(2.7-14.5 \%)$. By GA, stage III developed in: $\leqslant 27$ weeks, $7.1 \% \quad(25.0-50.6 \%)$; $28-31$ weeks, $0.9 \%(1.8 \%-10.9 \%)$. The low incidence of stage III disease in Birmingham between 1989 and 1995 serves to highlight that even now there are significant differences between centres; indeed the incidence of severe disease is much less than in another study undertaken by the same observer in a different population. ${ }^{9}$ Several factors contribute to this variability, including neonatal survival, standard of care and ethnic mix. While it is recognised that diagnoses by individual clinicians can vary ${ }^{20}$ this is less likely for severe disease than mild ROP. Our data show that stage III babies requiring treatment were less mature than those who did not, but the differences in GA of 0.4 weeks and BW of $63 \mathrm{~g}$ are not significant in clinical practice terms.

The current indication for treatment is 'threshold' disease - stage III ROP extending over at least 5 continuous clock-hours, or for at least 8 cumulative clock-hours - at which stage the risk of blindness is around $50 \%{ }^{1}$ Although treatment of threshold ROP has been shown to reduce unfavourable outcome (vision worse than $6 / 60$ ) from $50.6 \%$ to $31.9 \%$ in zone 2 disease at 1 year, ${ }^{21}$ at $3 \frac{1}{2}$ years the difference was $57.5 \%$ compared with $46.6 \%{ }^{22}$ At $5 \frac{1}{2}$ years, while fewer treated than control eyes were blind ( $31.5 \%$ vs $48.0 \%)$ slightly more control than treated eyes $(17 \%$ vs $13 \%)$ had normal vision $(6 / 12$ or 
better). ${ }^{23}$ The results for zone 1 disease are even less encouraging. ${ }^{21}$ Thus, while the value of treatment be it cryotherapy or laser - is proven in severe ROP, the outcome still falls short of the ideal. Consequently the sense of delaying treatment until there is a $50 \%$ risk of blindness must be questioned. Of course, this must be balanced against the risk of treating babies who would anyway undergo spontaneous resolution. ${ }^{24}$ It is entirely appropriate, therefore, that ophthalmologists question the current indications for treatment. For ROP which does not progress beyond stage I or II, spontaneous resolution without visually disabling sequelae occurs in all cases, ${ }^{4,5}$ and clearly intervention is not an issue. The dilemma centres, therefore, on determining which stage III eyes require treatment. The current indication for treatment takes into account only total clock-hour involvement of stage III, giving equal weighting to all regions of the retina, and does not account for the possibility that retinopathy may have a greater propensity for affecting vision in one part of the retina than another. ${ }^{24}$ This debate will almost certainly develop further in the immediate future, but for these reasons we have, in this study, focused on any stage III disease.

Over the past decade or so, many protocols for screening have been devised. 3,16,18,19,25-34 There have been advocates for basing screening inclusion criteria on $\mathrm{BW},{ }^{25,31,34,35} \mathrm{GA}^{18}{ }^{18}$ or, because of discrepancies between the two, both BW and GA..$^{3.30,32,33,36}$ Several studies have identified severe ROP in babies born outside the $\leqslant 1250 \mathrm{~g} \mathrm{BW}$ criterion which was used for the CRYO-ROP Study. ${ }^{1,16,19,30,35,36}$ Thus in Edinburgh $^{19} 1$ of 47 , East Midlands ${ }^{9} 2$ of 27 and the London study ${ }^{16} 2$ of 35 , were born heavier than $1250 \mathrm{~g}$ but had GAs $\leqslant 29$ weeks. If $\mathrm{GA} \leqslant 29$ weeks alone were considered as the single inclusion criterion, three studies identified babies with stage III disease born after 29 weeks but with BW $\leqslant 1250$ g: 1 of 47 from the Edinburgh study, ${ }^{19} 2$ of 27 from the East Midlands ${ }^{15}$ and 1 of 9 from New Zealand. ${ }^{14}$ In our study, 2 of 39 stage III babies would have fallen outside a single inclusion criterion of either $\leqslant 1250 \mathrm{~g} \mathrm{BW}$ or $\leqslant 29$ weeks GA $(1375 \mathrm{~g} /$ 29 weeks and 31 weeks/930 g respectively), although both would have been identified using BW and GA criteria together. We have attempted to determine whether any baby in these publications would have remained unidentified if $\mathrm{BW}$ and GA were combined. While this was not possible in each article, in an Australian study, ${ }^{35}$ two babies with stage III would have been missed (1290 g and 33 weeks, $1435 \mathrm{~g}$ and 32 weeks), and in Denmark ${ }^{30}$ blindness developed in $16>1250 \mathrm{~g} \mathrm{BW}$ and $6 \geqslant 31$ weeks GA (ROP grading not provided). So far we have concentrated on the routine screening of preterm populations meeting the inclusion criteria of a particular screening protocol. However, it is possible that there are certain babies, some of who may fall outside the criteria, who are particularly susceptible to developing severe ROP. One such high-risk group might be babies suffering severe ischaemic neurological insults. It is pertinent to note that an association between severe ROP and periventricular leucomalacia has been reported ${ }^{37}$ in 6 babies of which one was GA 30 weeks and BW $1440 \mathrm{~g}$. Recently, 3 babies of 32-33 weeks GA but $>2000$ g BW who experienced severe prenatal blood loss and subsequently underwent surgery with general anaesthesia developed ROP reaching stage III in 2, with one requiring cryotherapy. ${ }^{38}$ Clearly it would be inappropriate to widen the screening criteria to include all babies of BW $>2000 \mathrm{~g}$. This would increase the workload several-fold with little clinical benefit. However, such cases are important, because common aetiological factors may emerge - such as severe neurological injury or perinatal blood loss - which might identify babies falling outside the criteria and who require screening. To screen these additional at-risk babies would not pose a major additional workload.

Keith and Doyle ${ }^{35}$ predicted that the inclusion of $\mathrm{GA}$, in addition to $\mathrm{BW}$, as a criterion for screening would substantially increase workload. This expectation has not been realised; on the contrary, the combination of BW and GA would signficantly reduce the number of babies requiring screening.

It was particularly interesting to show how an alteration of the inclusion criteria might have enabled detection of all stage III babies whilst eliminating babies not requiring screening. Indeed, the effect of changing the inclusion criteria to $\leqslant 1250 \mathrm{~g} \mathrm{BW}$ or $\leqslant 29$ weeks GA would, in this study, have reduced the number of babies requiring screening by $30 \%$ (432) of 1429 , and even more examinations. In the East Midlands study, ${ }^{15} 54 \%$ fewer babies would have required screening, although the sole inclusion criterion was $\leqslant 1700 \mathrm{~g}$ $\mathrm{BW}$. It is not appropriate to attempt to alter national screening guidelines on the strength of a single study, because, as shown above, there are significant differences in the incidence of severe disease between neonatal populations. Furthermore, a few babies have developed severe ROP who fell outside these criteria. To achieve clinical effectiveness, periodic review of existing guidelines should be undertaken. The estimated UK population of infants born weighing $<1500 \mathrm{~g}$ each year is around 6600 , with the proportion of survivors increasing. ${ }^{32}$ This figure does not take into account those born $>1500 \mathrm{~g}$ but $<32$ weeks GA, which represented 224 babies (16\%) of this study. Nevertheless, nationally, introducing new inclusion criteria could save 2300 babies annually from an ophthalmic examination. Evidence does not support the recent proposal for the 
exploration of screening examinations to be undertaken by professions other than ophthalmologists, for babies of $>1500 \mathrm{~g} \mathrm{BW}^{39}$ We concur with Bancalari ${ }^{40}$ that these larger babies need not be screened at all and efforts should be directed to an appropriate examination of smaller babies.

If other UK centres confirm our finding that few stage III babies are likely to be missed using revised criteria of $\leqslant 1250 \mathrm{~g} \mathrm{BW}$ or $\leqslant 29$ weeks GA, then it would seem appropriate to reconsider guidelines for ROP screening. However, it is important that the clinical events placing larger babies at risk are identified (e.g. severe neurological insult or blood loss) so that clinical guidelines can be fine-tuned accordingly, ensuring efficient practice yet without inappropriately increasing the screening load.

We thank the neonatologists and nursing staff of the Birmingham Maternity, City, Sorrento, Heartlands, Good Hope and Solihull Hospital neonatal units for facilitating ROP screening.

Key words: Retinopathy of prematurity, Neonate, Screening, Criteria.

\section{REFERENCES}

1. Cryotherapy for Retinopathy of Prematurity Cooperative Group. Multicenter trial of cryotherapy for retinopathy of prematurity: preliminary results. Arch Ophthalmol 1988;106:471-9.

2. Javitt J, Dei Cas R, Change Y. Cost-effectiveness of screening and cryotherapy for threshold retinopathy of prematurity. Pediatrics 1993;91:859-66.

3. Report of a Joint Working Party: The Royal College of Ophthalmologists \& British Association of Perinatal Medicine. Retinopathy of prematurity: guidelines for screening and treatment. London, 1995.

4. Laws D, Shaw DE, Robinson J, Jones HS, Ng YK, Fielder AR. Retinopathy of prematurity: a prospective study: review at six months. Eye 1992;6:477-83.

5. Cryotherapy for Retinopathy of Prematurity Cooperative Group. The natural ocular outcome of premature birth and retinopathy: status at 1 year. Arch Ophthalmol 1994;112:903-12.

6. Dhillon B, Wright E, Fleck BW. Screening for retinopathy of prematurity: are a lid speculum and scleral indentation necessary? J Pediatr Ophthalmol Strabismus 1993;30:377-81.

7. Committee for the Classification of Retinopathy of Prematurity. An international classification of retinopathy of prematurity. Br J Ophthalmol 1984;68:690-7.

8. Committee for the Classification of Retinopathy of Prematurity. An international classification of retinopathy of prematurity. II. The classification of retinal detachment. Arch Ophthalmol 1987;105:906-12.

9. Ng YK, Fielder AR, Shaw DE, Levene MI. Epidemiology of retinopathy of prematurity. Lancet 1988;2:1235-8.

10. Keith CG, Kitchen WH. Retinopathy of prematurity in extremely low birthweight infants. Med J Aust 1984;141:225-7.

11. Reisner SH, Amir J, Shohat M, Krikler R, Nissenkorn I, Ben-Sira I. Retinopathy of prematurity: incidence and treatment. Arch Dis Child 1985;60:698-701.
12. Schaffer DB, Johnson L, Quinn GE, Weston M, Bowen FW. Vitamin E and retinopathy of prematurity. Ophthalmology 1985;92:1005-11.

13. Flynn JT, Bancalari E, Bachynski BN, Buckley EB, Bawol R, Goldberg R, Cassidy J, et al. Retinopathy of prematurity: diagnosis, severity and natural history. Ophthalmology 1987;94:620-9.

14. Schulenburg WE, Prendiville A, Ohri R. Natural history of retinopathy of prematurity. Br J Ophthalmol 1987;71:837-43.

15. Darlow BA. Incidence of retinopathy of prematurity. Arch Dis Child 1988;63:1083-6.

16. Acheson JF, Schulenburg WE. Surveillance for retinopathy of prematurity in practice: experience from one neonatal intensive care unit. Eye 1991;5:80-5.

17. Palmer EA, Flynn JT, Hardy RJ, et al. The Cryotherapy for Retinopathy of Prematurity Cooperative Group. Incidence and early course of retinopathy of prematurity. Ophthalmology 1991;98:1628-40.

18. Holmstrom G, Azazi M, Jacobson L, Lennerstrand GA. A population based, prospective study of the development of ROP in prematurely born children in the Stockholm area of Sweden. Br J Ophthalmol 1993;77:417-3.

19. Fleck BW, Wright E, Dhillon B, Millar GT, Laing IA. An audit of the 1995 Royal College of Ophthalmologists Guidelines for screening for retinopathy of prematurity applied retrospectively in one regional neonatal intensive care unit. Eye 1995;9:31-5.

20. Freedman SF, Kylstra JA, Hall JG, Capowski JJ. Plus disease in retinopathy of prematurity: photographic evaluation by an expert panel. Invest Ophthalmol Vis Sci 1995;36(Suppl):18.

21. Cryotherapy for Retinopathy of Prematurity Cooperative Group. Multicentre trial of cryotherapy for retinopathy of prematurity: one year outcome. Arch Ophthalmol 1990;108:1408-16.

22. Cryotherapy for Retinopathy of Prematurity Cooperative Group. Multicenter trial of cryotherapy for retinopathy of prematurity: $3 \frac{1 / 2}{2}$ year outcome structure and function. Arch Ophthalmol 1993;111: 339-44.

23. Cryotherapy for Retinopathy of Prematurity Cooperative Group. Multicenter trial of cryotherapy for retinopathy of prematurity: Snellen visual acuity and structural outcome at $5 \frac{1}{2}$ years after randomisation. Arch Ophthalmol 1996;114:417-24.

24. Fielder AR, Quinn GE. Evidently, evidence based. Br J Ophthalmol 1996;80:273-4.

25. Brown DR, Biglan AW, Stretavsky MAM. Screening criteria for the detection of retinopathy of prematurity in patients in a neonatal intensive care unit. J Pediatr Ophthalmol Strabismus 1987;24:212-4.

26. Tan KEWP, Cats BP. Timely incidence of examination for acute retinopathy of prematurity (ROP) and its consequence for the screening strategy. Am J Perinatol 1989;6:337-40.

27. Biglan AW, Cheng KP, Brown DR. Update on retinopathy of prematurity. Int Ophthalmol Clin 1989;29:1-9.

28. Schaffer DB. Update on retinopathy of prematurity: the examination guidelines. Semin Ophthalmol 1990;5: 100-6.

29. Darlow BA, Clemett RS. Retinopathy of prematurity: screening and optimal use of the ophthalmologist's time. Aust NZ J Ophthalmol 1990;18:41-6. 
30. Fledelius HC, Ros 'nberg T. Retinopathy of prematurity. Where to set he screening limits? Recommendations based on two Danish surveys. Acta Pediatr Scand 1990;79:906-10.

31. Thouvenin D, Legavre L, Bourdiol AM, Arne JL. Retinopathie de la prematurité. J Fr Ophtalmol 1992; 15:191-7.

32. Fielder AR, Levene MI. Screening for retinopathy of prematurity. Arch Dis Child 1992;67:860-7.

33. Clark DI, O'Brien C, Weindling AM, Saeed M. Initial experience of screening for retinopathy of prematurity. Arch Dis Child 1992;67:1233-6.

34. Mattern AB, Pohlandt F. The optimal screening point for assessing the therapy-relevant stages of retinopathy of prematurity. Ophthalmologe 1994;91:343-7.

35. Keith CG, Doyle LW. Retinopathy of prematurity in infants weighing 1000-1499 g at birth. J Paediatr Child Health 1995;31:134-6.
36. Fielder AR, Shaw DE, Robinson J, Ng YK. Natural history of retinopathy of prematurity: a prospective study. Eye 1992;6:233-42.

37. Ng YK, Fielder AR, Levene MI, Trounce JQ, McLellan N. Are severe acute retinopathy of prematurity and severe periventricular leucomalacia both ischaemic insults? Br J Ophthalmol 1989;73:111-4.

38. Jandeck C, Kellner U, Kossel H, Bartsch M, Versmold HT, Foerster MH. Retinopathy of prematurity in infants of birth weight $>2000 \mathrm{~g}$ after haemorrhagic shock at birth. Br J Ophthalmol 1996;80:728-31.

39. Flynn JT, Sola A, Good WV, Phibbs RH. Screening for retinopathy of prematurity: a problem solved? Pediatrics 1995;95:755-7.

40. Bancalari E. A neonatologist's viewpoint. Pediatrics 1995;95:763. 